\title{
Energy Consumption Optimization using Clustering in Mobile Ad-Hoc Network
}

\author{
Jyotika Chandel \\ MTech. Scholar \\ SIRT, Bhopal \\ M.P, India
}

\author{
Navneet Kaur \\ Professor \\ Dept. of ECE \\ SIRT, Bhopal \\ M.P, India
}

\begin{abstract}
In this In MANET (Mobile Ad hoc Network) nodes are not fixed on one position that's why proper communication is very critical issue. The nodes in MANET depend on battery for communication. Therefore energy efficiency is an important design deliberation to extend the lifetime of networks. The cluster based approach provides the efficient communication in the form of group. The nodes in Mobile ad hoc network, communicate with each other in the cluster or outside the cluster. In this research work we proposed the cluster based approach along with multipath routing in MANET. The Low Energy Adaptive Clustering Hierarchy $(\mathrm{LEACH})$ is energy efficient protocol and the cluster based approach works on the basis of higher energy level. In this paper we compare the performance of multipath AOMDV (Ad hoc On-demand multipath Distance Vector) and AOMDV with LEACH protocol. In normal multipath routing the route selection is based on the shortest path that's why energy consumption is also more due to retransmission in case of link breakage. This problem is sort out in proposed approach by selecting higher energy level of nodes and the whole communication is based on Cluster Head $(\mathrm{CH})$. The cluster Head $(\mathrm{CH})$ selection is based on higher energy level of nodes in the cluster and also improves the energy utilization in network. The performance of proposed protocol is better than previous work, and provides reliable communication.
\end{abstract}

\section{Keywords}

MANET, AOMDV, Residual energy, LEACH Protocol, Clustering, Multipath Routing.

\section{INTRODUCTION}

A Mobile Ad hoc Network (MANET) is a self-configuring infrastructure less network of mobile devices connected by wireless [1]. In a MANET each and every node is free to move in any direction, and hence change its links to other nodes frequently. All the nodes cooperatively maintain network connectivity without the aid of any fixed infrastructure units. Each node has a routing function whereby it communicates by forwarding packets via intermediate nodes. If two nodes are within the transmission range of each other, they communicate directly. Otherwise, other nodes are needed to forward their packets. MANET is characterized by non-restricted mobility and easy deployment, which makes them very promising. Ad hoc networks have become increasingly relevant year due to their potential application in battlefield, military, and emergency disaster relief etc. A goal of MANET is to broaden mobility into the area of autonomous, mobile and wireless domains, whereas a set of nodes from the network routing infrastructure in ad-hoc manner.In ad hoc networks, nodes communicate with each other by way of radio signals, which are broadcast in nature. Broadcast is a unique case of multicast, wherein all nodes in the network should get the broadcast message. Multicasting is a communication process in which the transmission of packets (message) is initiated by a single user and the message is received by one or more end users of the network. Multicasting in wired and wireless networks has been advantageous and used as a vital technology in many applications such as audio/ video conferencing, corporate communications, collaborative and groupware applications, distance learning, stock quotes, distribution of software, news and etc [2]. It might not be possible to recharge / replace a mobile node that is powered by batteries during a mission. The inadequate battery lifetime imposes a limitation on the network performance [3]. To take full advantage of the lifetime of nodes, traffic should be routed in a way that energy consumption is minimized. In recent years, various energy efficient routing protocols have been proposed. These protocols have unique attributes and utilize different recovery mechanisms on energy consumption.

\section{LEACH PROTOCOL}

Low Energy Adaptive Clustering Hierarchy (LEACH) may be a special energy efficient protocol for WSN [4]. LEACH uses cluster based mostly technique. During a network there are several sensors each one sensor conjointly decides the cluster head in every round. LEACH uses random method to distribute the energy load between sensors. To choose cluster head $(\mathrm{CH})$ in each round we have a tendency to decide a threshold. During this method the node that is now cluster head work as a base station. To compress data it uses local data fusion to compress data that is distributed from cluster to cluster head or base station. Compression is completed to extend the time period and to scale back the energy dissipation. LEACH conjointly cut back redundancy by data fusion technique. In LEACH as a result of the cluster head node have additional work to try and do like data receive, send, to manage redundancy or data fusion therefore energy utilized by cluster head is over alternative nodes. Therefore it's build it a lot of attainable to die. LEACH is an energy economical protocol work in wireless detector network. It's supported the construct of hierarchal routing; the main aim of LEACH is to extend the life time of the network. 


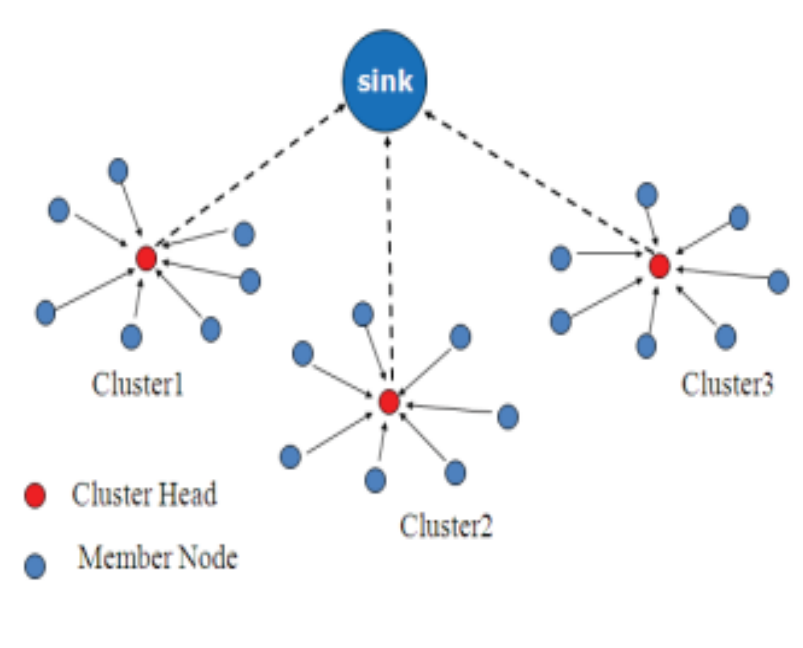

Fig. LEACH Example

\section{MULTIPATH ROUTING}

Please Multipath routing has been explored in several different contexts. Traditional circuit switched telephone networks used a type of multipath routing called alternate path routing. In alternate path routing, each source node and destination node have a set of paths (or multi-paths) which consist of a primary path and one or more alternate paths. Alternate path routing was reposed in order to decrease the call blocking probability and increase overall network utilization.

\subsection{AOMDV Procedure}

The AOMDV uses the basic AODV route construction process. In this case, some extensions are made to create multiple link-disjoint paths. The main idea in AOMDV is to compute multiple paths during route discovery. It consists of two components:

- A route update rule to establish and maintain multiple loop-free paths at each node.

- A distributed protocol to find link-disjoint paths.

In AOMDV [5] each RREQ, respectively RREP arriving at a node defines an alternate path to the source or destination. Just accepting all such copies will lead to the formation of routing loops. In order to eliminate any possibility of loops, the "advertised hop count" is introduced. The advertise hop count of a node $i$ for a destination node $d$ represents the maximum hop count of the multiple paths for node $d$ available at $i$. The protocol only accepts alternate routes with hop count lower than the advertised hop count, alternate routes with higher or the same hop count are discarded. The advertised hop count mechanism establishes multiple loop-free paths at every node. These paths still need to be disjoint. We use the following:

When a node $\mathrm{S}$ floods a RREQ packet the network, each RREQ arriving at node intermediate node I via a different neighbour of S, or S itself, define a node-disjoint path from I to $\mathrm{S}$. In AOMDV this is used at the intermediate nodes. Duplicate copies of a RREQ are not immediately discarded. Each packet is examined to see if it provides a node-disjoint path to the source. For node-disjoint paths all RREQs need to arrive via different neighbours of the source. This is verified with the first hop field in the RREQ packet and the first hoplist for the RREQ packets at the node.At the destination a slightly different approach is used, the paths determined there are link-disjoint, not node-disjoint. In order to do this, the destination replies up to $\mathrm{k}$ copies of the RREQ, regardless of the first hops. The RREQs only need to arrive via unique neighbours.

\section{PREVIOUS WORK}

Various research works has been done for energy efficiency in network. In this section we focus on previous work that is done on this field.

Amit Munjal et al. [6], proposed a new energy efficient clustering based approach, that attempts to equalize the energy consumption between different clusters in wireless sensor network. The proposed protocol is simulated and compared with an existing LEACH protocol.

Sahar Fattahi et al. [7] proposed two algorithms LEACH-C and IDEC(Improved Definite clustering Protocol). In this research, it is decided that in order for improving the results, the LEACH-C protocol, which excels the performance of the LEACH protocol, to be combined with the protocol IDEC (Improved DEC). The protocol ICED \& LEACH-C saves energy. As we know, decreased energy consumption increases lifetime of the WSN network.

DanWu, Gai-Yun Wang \& Xiao-Long Li [8], proposed a distributed TDMA scheduling protocol based on coloring algorithm (TDMA-CA), which uses spatial reuse of transmission channel. It allocates different colors for conflict nodes in the networks, and arranges distinct slots for data transmission for each color by TDMA scheduling. Simulation results show that TDMA-CA is better than SMAC (Static MAC) in terms of both energy efficient and latency in WSN.

A.S.Salunkhe et al. [9], proposed routing scheme which is a position based routing approach which incorporates Dynamic Selection of the Gateway nodes to reduce the number of control packets flooded in the network required for efficient routing of data packets. This work presents routing algorithm, cluster algorithm and simulation environment and scheme used to performance evaluation using cluster based routing protocol in MANET.

Baisakh \& Nileshkumar R. Patel [10], proposed ESSDSR (Energy saving and survival routing protocol) which acts not only as an energy efficient routing protocol but also shows an energy survival instinct in mobile ad hoc network (MANET). The proposed protocol the node's life time has been improved as compared to traditional DSR with a higher ratio and also evaluated the life time of the networks

Gopi Saminathan Arumugam et al. [11], proposed EELEACH (Energy Efficient- LEACH) yields better performance than the existing energy-balanced routing protocol (EBRP) and LEACH Protocol in terms of better packet delivery ratio, lesser end-to-end delay and energy consumption. It proves that the proposed EE-LEACH can improve the network lifetime.

\section{PROBLEM STATEMENT}

Mobile nodes in MANET use the more battery power while transmitting and receiving the data therefore there is more energy consumption in data transfer. The new innovative idea of energy efficient method is required thus the problem of energy consumption is solved using multipath routing with clustering scheme, energy consumption controlled in the network. MANET has no fixed infrastructure and nodes of the mobile ad hoc network are typically powered by batteries. So node stops functioning when the battery drains. When battery power off node will be die and established link break so because of that more problem created like packet will be 
dropped, data does not transmitted properly, retransmission of the data occurs. Thus Energy efficiency is an important consideration in such an environment. Since energy-saving techniques aimed at minimizing the total energy consumption of all nodes in the group (minimize the number of nodes used to establish connectivity, minimize the overhead and so on) and at maximizing the life span should be considered.

\section{PROPOSED SCHEME}

Proposed LEACH improves the performance of LEACH algorithm in terms of energy consumption and packet delivery ratio in real time networks. Every sensor node does not have data to send all the time. The data is available in a random order. The mobile nodes may be event driven, so that data may be available only when they sense any event. The proposed method is optimized by utilizing the slots belonging to the node that having no data to send. This improved method turns free time slots into useful slots without making any changes in the prescribed TDMA schedule. It will also reduce the waiting time for mobile nodes because now there is a chance that sensor nodes can get more than one time slot per frame to transfer their data. Moreover, Multi-hop routing is used to transfer data to the receiver because when data packet is sent directly by $\mathrm{CH}$ to the destination or next cluster head then due to increased distance in between them it can lead to increase path loss exponentially due to multipath fading. The methodology adopted in proposed method is like traditional LEACH but with improvement. The scheme consists of following two main phases: cluster setup phase and steady state transmission phase. Cluster set-up phase includes cluster head selection and cluster formation. Steady state phase includes transmission of data from mobile nodes to $\mathrm{CH}$ and then data transmission from $\mathrm{CH}$ to next cluster head.

\subsection{Cluster Set-Up Phase}

As its name suggests, the cluster setup phase sets up the clusters. Before cluster formation, cluster heads must be selected. Proposed algorithm divide the working of this phase in following two sub phases:

\section{A)Cluster Head Selection}

At the initial phase of each round, each node advertises its probability for becoming the cluster head $(\mathrm{CH})$ (depending upon its current energy level). Mobile nodes ( $\mathrm{k}$ for every round) with larger probabilities are chosen as the Cluster Heads. Cluster Heads broadcast an advertisement message using CSMA MAC protocol. Cluster Head advertisement message contains two fields node's id and header part. Node's ID is a number identifying every node uniquely and header part having control information. Based upon the higher energy strength every non-Cluster Head node discovers it's Cluster Head for this round. Cluster heads changes randomly over time in order to maintain a balance of energy dissipation in between the nodes. This decision is taken by the node; choosing a random number between 0 and 1 . The sensor node becomes a cluster head if the number is less than threshold for the current round.

\section{2) Cluster Formation}

Based upon the received signal strength of $\mathrm{CH}$ advertisement message, each non-Cluster Head transmits a cluster joinrequest message back to its chosen Cluster Head $(\mathrm{CH})$ using a CSMA MAC protocol. Join Request message contains three fields. Node's ID which is a number identifying each node uniquely. Cluster head ID which is the number identifying each cluster head uniquely and header which contain control information such as source and destination address. Cluster
Head node creates a TDMA schedule for each non-CH node for data transmission coordination inside the cluster. After the schedule is being created, the cluster-head broadcasts the created schedule to all nodes inside its cluster. When the mobile nodes receive the message, they arrange their duty cycle of radio communication modules to set turned on or off as per the allotted time slots. Establish multipath for enhance routing performance.

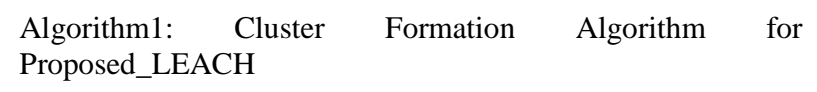

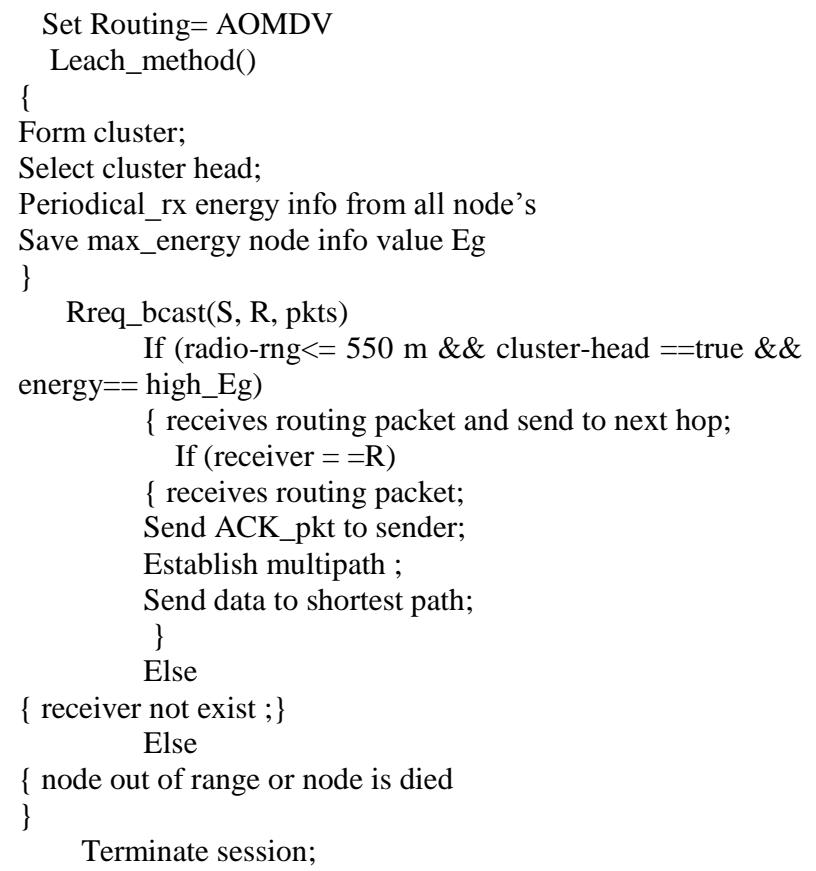

\section{B.Data Transfer Models}

The process of data transmission is done in steady state phase. Proposed algorithm divide the working of this phase in following two sub phases. There are two types of data transfer:

- Data transfer to a coordinator from a device

- Data transfer from a coordinator to a device

\section{1) Data Transfer to a Coordinator}

When a device decides to transmit data to the coordinator, the device synchronizes its clock on a regular basis and transmits the data to the coordinator using the CSMA-CA method. It has been assumed that the transmission does not occur during a GTS. The coordinator may acknowledge the reception of the date only if it is requested by the data transmitter.

Steady state phase pseudo code of proposed leach Input: Output of steady phase

Output: Allocation of slots to the sensor nodes according to data they have to send

1. begin

2. if $(\mathrm{CH}(\mathrm{s})=$ True $)$ then

3. Transmit (IDi, Data pck); //ID is node number

4. Aggregate (IDi, Data pck);

5. S to R (IDi, Data pck); // Part of Data Transfer Phase through Multipath

6. else 
7. for ( $\mathrm{i}=1$; $\mathrm{i}<$ total_slots; $\mathrm{i}++)$ // time slot according to TDMA approach

8. \{

9. if $($ My_time_slot $=$ True $\& \&$ DataToSend $=$ true $)$ then

10. Trans_to_CH(IDi, Data pck); // Establish multipath for data in between sender and receiver.

11. else

12. if (Data_To_Send $=$ false $)$ then

13. TransfeSlotToNextNode(IDi)

14. end if

15. Sleep_mode $=$ true;

16. end if

17. $\}$

18. end if

19. TransfeSlotToNextNode(IDi);

20. My_time_slot $=$ false;

\section{SIMULATION AND RESULTS}

Please NS2 is an open-source event-driven simulator designed specifically for research in computer communication networks. The simulator we have used to simulate the ad-hoc routing protocols in the Network Simulator 2 (ns2) from Berkeley [20].

\subsection{Simulation Parameter}

Table 1 represents the simulation parameters to make the scenario of routing protocols. The detailed simulation model based on network simulator-2 (ver-2.31) [12], is used in the evaluation. The NS instructions can be used to define the topology structure of the network and the motion mode of the nodes, to configure the service source and the receiver etc.

Table 1 Simulation parameters

\begin{tabular}{|l|c|}
\hline Simulator Used & NS-2.34 \\
\hline Number of nodes & 100 \\
\hline Dimension of simulated area & $800 \mathrm{~m} \times 600 \mathrm{~m}$ \\
\hline Routing Protocol & AOMDV \\
\hline Simulation time & 500 sec. \\
\hline Traffic type & CBR $(3 \mathrm{pkts} / \mathrm{s})$ \\
\hline Packet size & 512 bytes \\
\hline Number of traffic connections & 7 \\
\hline $\begin{array}{l}\text { Node movement at maximum } \\
\text { Speed }\end{array}$ & random $(20 \mathrm{~m} / \mathrm{s})$ \\
\hline Transmission range & $250 \mathrm{~m}$ \\
\hline Transmission Energy & 1.5 Joule \\
\hline Receiving Energy & 1 Joule \\
\hline Ideal Energy & .01 Joule \\
\hline Sense Power & .175 Joule \\
\hline
\end{tabular}

\subsection{Performance Parameter}

There are following different performance metrics that have been considered to make the comparative study of these protocols through simulation.

1) Routing overhead: This metric describes how many routing packets for route discovery and route maintenance need to be sent so as to propagate the data packets.

2) Throughput: This metric represents the total number of bits forwarded to higher layers per second. It is measured in bps

3) Residual Energy: The remaining energy of the network after end of the process is called residual energy. It should be more for better performance.

\subsection{Results}

In this section the performance of normal AOMDV routing and proposed AOMDV with LEACH is presented and it is analyzed that the performance of proposed LEACH protocol is better in term of energy utilization and routing performance in dynamic network.

\section{1) Overhead Analysis}

The number of sender nodes in network first flooded the routing packets in network and after connection establishment; sender is sending data to receiver. If the retransmission in network is happening again and again due to connection loss then in that condition, it is not possible to utilize limited battery power of nodes. The routing packets quantity in proposed cluster based communication is only 40000 pks but in normal routing this quantity is about four times more than the proposed scheme, which shows the heavy depletion in energy due to reestablishment of connection. The LEACH protocol with AOMDV multipath routing improves the routing technique so as also the routing packets flooding are minimized in dynamic network. In group communication the responsibility of Cluster Head $(\mathrm{CH})$ is more that's why the $(\mathrm{CH})$ energy is higher than the all group members in dynamic network.

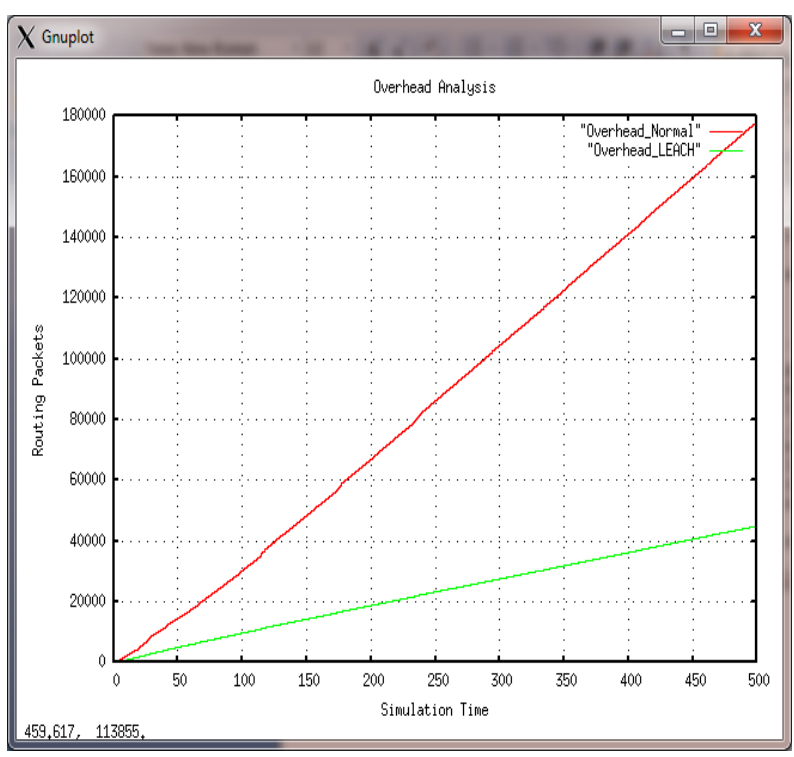

Fig.2 Overhead Performance Analysis

\section{2) Throughput Performance Analysis}

The successful data receiving in network is measured through maximum number of packets received at destination. In throughput analysis actually performance is measured through number of bits but also in general it is receiving of data or sending of data in per unit of time. That means in unit time, measures the performance of network. In this network the throughput performance of cluster based LEACH routing is much better than normal energy routing. The main reason of better communication is group based routing using clustering. 


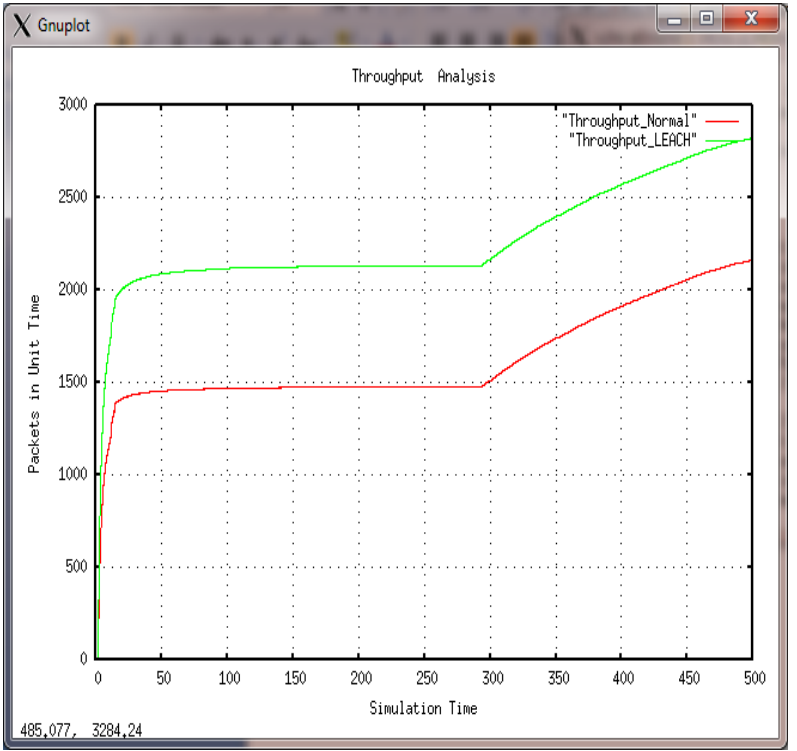

Fig.3 Throughput Analysis

\section{4) Residual Energy}

The energy consumption on different time instance is mentioned in this graph. The remaining energy of nodes determines the survival of nodes in the network. The performance of nodes residual energy is evaluated in different time instance like 100, 200, 300, 400 and 500 seconds. The energy consumption in normal multipath routing and the cluster based communication is consumes less amount of energy therefore, energy utilization is improved and enhance routing performance.

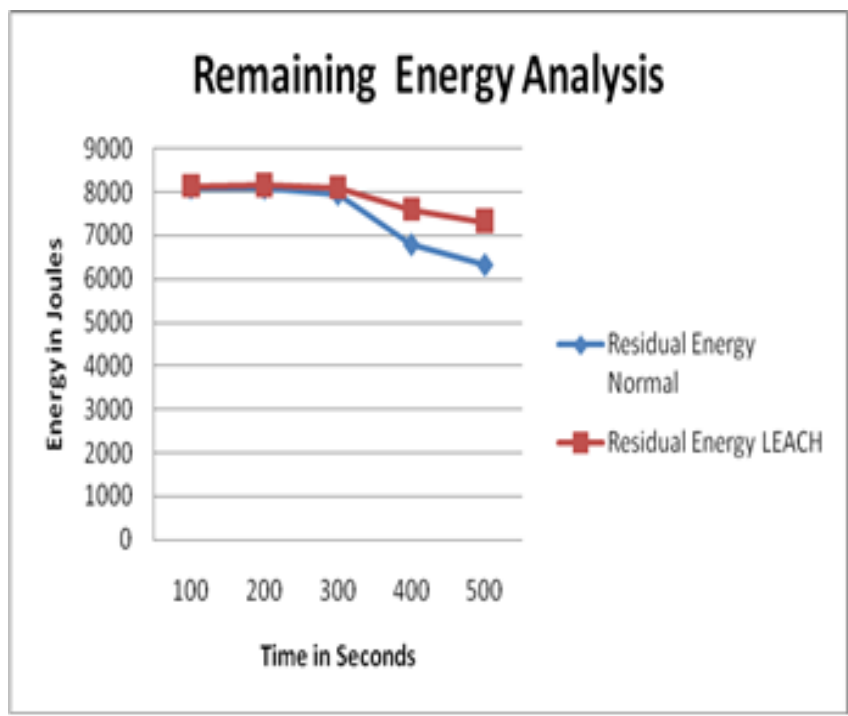

Fig.4 Energy Analysis in Different Time

\section{CONCLUSION AND FUTURE WORK}

Recharging or replacing batteries will often not be possible instantly in dynamic network. In ad hoc a network, sender nodes does not communicate on its own, it still normally forwards routing packets for others for connection establishment and then from establish route sender is send data to destination. The proper communication without link breakage reduces the battery power consumption. In this paper we proposed energy efficient LEACH protocol with multipath routing for improved energy utilization and network performance. The proposed energy efficient scheme is to select the node as the cluster head having higher energy in the group. The higher energy of $\mathrm{CH}$ is capable to handle the request of cluster members efficiently and also provides reliable communication in network. Further, the balance among established multiple paths based on the energy usage in a path selection. The proposed LEACH routing scheme show great potential and evenly reduces energy consumption and provides strong path. The cluster based communication is efficient because communication in the cluster or outside the cluster is based on $\mathrm{CH}$ and need not to interact with other nodes for finding destination or sending data to destination. The performance of LEACH is better than energy based multipath routing in term of throughput, PDR and Routing overhead. The energy consumption in network is also reduced that is prolonging the network life time.

The energy is the limited resource of communication in dynamic network. There are many reasons of energy consumption in network like congestion, collision and any type of attacker existence in network. In future we proposed the congestion control scheme can be implemented to reduce data loss and utilizes energy consumption in communication.

\section{REFERENCES}

[1] C. S. R. Murthy and B. S. Manoj, "Ad hoc Wireless Networks: Architectures and Protocols", Prentice Hall, 2004.

[2] D. Helen and D. Arivazhagan, "Applications, Advantages and Challenges of Ad Hoc Networks", Journal of Academia and Industrial Research (JAIR), pp553-457 Volume 2, Issue 8 January 2014.

[3] Gurpinder singh, Jaswinder Singh, "MANET", "A Study of Challenges and Routing Principles", International Journal of Advanced and Innovative Research, ISSN: 2278-7844 Vol. 1, Issue 1, 2012.

[4] Vishnu Kumar Sharma and Dr. Sarita Singh Bhadauria "Mobile Agent Based Congestion Control Using AODV Routing Protocol Technique For Mobile Ad-Hoc Network" International Journal of Wireless \& Mobile Networks (IJWMN) Vol. 4, No. 2, April 2012.

[5] D.Joshi Radhika, P.Rege Priti,"Energy Aware Routing in Ad Hoc Networks", 6th WSEAS International Conference on Circuits, Systems, Electronics, Control \& Signal Processing, Cairo, Dec 29-31, 2007.

[6] M. Tubaishat, S. Madria, "Sensor Networks: An Overview “, IEEE Potentials, April/May 2003.

[7] Shio Kumar Singh, M P Singh, D K Singh, “A Survey of Energy-Efficient Hierarchical Cluster-Based Routing in Wireless Sensor Networks", International Journal of Advanced Networking and Applications Volume: 02, Issue: 02, pp. 570-580, 2010.

[8] Yuan P, Bai Y and Wang H, "A Multipath EnergyEfficient Routing Protocol for Ad hoc Networks", In Proceedings of International Conference on Communications, Circuits and Systems, Vol. 3, pp. 1462 $-1466,2006$.

[9] Prashant Krishan, "Comparison and Performance Analysis of Dynamic and Static Clustering Based Routing Scheme in Wireless Sensor Network", International Journal of Advanced Research in Computer and Communication Engineering, Vol. 2, Issue 4, April 2013. 
[10] M. Marina and S. Das, "On-demand Multipath Distance Vector Routing in Ad Hoc Networks", in Proceedings of the International Conference for Network Protocols (ICNP), Riverside, Nov. 2001.

[11] Mohammed Tarique, Kemal. E.Tepe, Sasan Adibi, Shervin Erfani, "Survey of multipath Routing Protocols for Mobile Ad hoc Networks", Journal of Network and Computer Applications, Pages 1125-1143, Volume 32 Issue 6, November, 2009.

[12] Qing Wu and Yixin Yan, "LEACH Routing Protocol Based on Wireless Sensor Networks", International Journal of Future Generation Communication and Networking Vol.7, No.5, pp. 251-258, 2014

[13] Sahar Fattahi \& Amir Najafi,"Clustering in Wireless Sensor Networks using IDEC-LEACH-C Hybrid Energy Efficient Protocol", International Journal of Computer Applications (0975 - 8887) Volume 141 - No.4, May 2016.
[14] DanWu, Gai-Yun Wang \& Xiao-Long Li, "Distributed TDMA Scheduling Protocol Based on conflict-free For Wireless Sensor Networks", IEEE International Conference on Intelligent Computing and Integrated Systems (ICISS), 2010.

[15] A.S.Salunkhe, Dr.S.V.Sankpal,"Performance Evaluation Using Cluster Based Routing Protocol for MANET", (IJAIEM) Volume 2, Issue 1, January 2013.

[16] Baisakh \& Nileshkumar R. Patel,"Energy Saving and Survival Routing Protocol for Mobile Ad Hoc Networks" International Journal of Computer Applications (0975 888) Volume 48- No.2, June 2012.

[17] Gopi Saminathan Arumugam and Thirumurugan Ponnuchamy, "EE-LEACH: development of energyefficient LEACH Protocol for data gathering in WSN" DOI 10.1186/s13638-015-0306-5 Springer journal, 2015

[18] The Network Simulator $\quad-\quad$ ns-2 http://www.isi.edu/nsnam/ns/ 